\title{
Catalyst stabilization by stoichiometrically limited layer-by-layer overcoating in liquid media
}

\author{
Florent Héroguel ${ }^{1}$, Benjamin P. Le Monnier ${ }^{1}$, Kristopher S. Brown, Juno C. Siu, \\ Jeremy S. Luterbacher*
}

Laboratory of Sustainable and Catalytic Processing, Institute of Chemical Sciences and Engineering, École Polytechnique Fédérale de Lausanne (EPFL), CH-1015 Lausanne, Switzerland

\section{A R T I C L E I N F O}

\section{Article history:}

Received 20 April 2017

Received in revised form 26 June 2017

Accepted 3 July 2017

Available online 4 July 2017

\section{Keywords:}

$\mathrm{Al}_{2} \mathrm{O}_{3}$

Biomass conversion

Furfural

Catalyst stability

Sol-gel

\begin{abstract}
A B S T R A C T
The use of metal oxide overcoats over supported nanoparticle catalysts has recently led to impressive improvements in catalyst stability and selectivity. The deposition of alumina is especially important for renewable catalysis due to its robustness in liquid-phase conditions. However, there are limited reports of work on alumina deposition and stabilization that goes beyond atomic layer deposition (ALD). Here, we present a layer-by-layer deposition technique for the controlled formation of conformal alumina overcoats in the liquid phase. This technique is easy to perform in common wet chemistry conditions. Alternated exposure of the substrate to stoichiometric amounts of aluminum alkoxide and water in liquidphase conditions leads to the formation of a porous overcoat that was easily tunable by varying synthesis parameters. The deposition of $60 \mathrm{Al}_{2} \mathrm{O}_{3}$ layers onto $\mathrm{Al}_{2} \mathrm{O}_{3}$-supported copper nanoparticles suppressed irreversible deactivation during the liquid-phase hydrogenation of furfural - a key biomass-derived platform molecule. The porous overcoat leads to highly accessible metal sites, which significantly reduces the partial site blocking observed in equivalent overcoats formed by ALD. We suggest that the ease of scalability and the high degree of control over the overcoat's properties during liquid-phase synthesis could facilitate the development of new catalyst overcoating applications.
\end{abstract}

(C) 2017 Elsevier B.V. All rights reserved.

\section{Introduction}

Stability has a huge influence on the industrial viability of catalytic materials. Catalyst deactivation incurs significant costs due to the need for material regeneration and eventual replacement, and therefore has been a major focus in petrochemical research [1]. Reversible deactivation is often due to coverage of the active site by chemical binding (e.g. poisoning) or coverage of the active site (e.g. coking) [1,2]. These forms of chemical deactivation can often be reversed by thermal treatment such as calcination with air. Irreversible deactivation is much more costly and is often due to physical or thermal processes [2,3]. Prominent irreversible deactivation mechanisms include support collapse, and coalescence or leaching of metal nanoparticles. Coalescence refers to the loss of active surface by metal nanoparticle aggregation through Ostwald ripening or sintering. Leaching refers to metal dissolution and occurs principally in solution.

\footnotetext{
* Corresponding author.

E-mail address: jeremy.luterbacher@epfl.ch (J.S. Luterbacher).

1 These authors contributed equally.
}

Because most fossil-derived molecules are volatile, refineries largely rely on gas phase catalysis. The shift to renewable substrates often involves liquid-phase catalytic processing. Such conditions are required because biomass-derived molecules usually have low volatility and are produced by biomass depolymerization or biological transformation in dilute aqueous or solvent streams [4-6]. Irreversible deactivation, including by sintering and leaching, tends to be more pronounced in liquid-phase conditions, which makes catalyst stability even more critical $[1,2,7]$.

Catalyst overcoating by deposition of a metal oxide layer is a promising method to curtail irreversible deactivation. Several overcoating methods have been shown to protect metal nanoparticles against thermal deactivation, and in some cases, coking. These methods include the use of atomic layer deposition (ALD) of alumina [8-10] and silica deposition by sol-gel [11-13]. Atomic layer deposition of alumina involves the subsequent exposure of the substrate to reactive alumina precursors or water in near-vacuum conditions. Each cycle leads to the deposition of an atomically thick layer of alumina, which allows very accurate control of the overcoat's thickness. However, the near-vacuum conditions lead to high set-up costs and limit scale-up possibilities. Furthermore, ALD conditions lead to the formation of a dense overcoat, which completely blocks access to the metal. Catalytic activity is only recovered after 
a calcination step that crystallizes and cracks the overcoat [10]. However, even after calcination, access to the metal is typically significantly reduced. In comparison, overcoating with $\mathrm{SiO}_{2}$ by solgel chemistry is low-cost and easy to scale up. Although the degree of control does not match ALD's, the overcoat's thickness can be kinetically controlled within a few nm due to the low reactivity of siloxide precursors $[14,15]$. The utility of silica overcoating is nevertheless limited by silica's low stability in high temperature liquid-phase conditions, which lead to rapid structural collapse [16].

Alumina is much more resistant in liquid-phase conditions, but the reactivity of its alkoxy precursors make its deposition difficult to control kinetically. Because of this, there are few practical liquidphase synthesis methods for producing alumina overcoats. Instead, attempts to deposit alumina using sol-gel techniques have focused on mimicking the layer-by-layer approach and purge sequences of ALD. Such methods have been reported for several oxides including titania [17-19], alumina [20,21] and zirconia [21] and involve the subsequent exposure of the catalyst to metal precursor and aqueous solutions. Multiple washes are performed between exposures to avoid precipitation of material off the surface. The powder is then dried and calcined to obtain a single molecular layer of oxide. These steps are repeated multiple times to achieve a conformal overcoat. This method is precise but extremely time-consuming, and its multiple steps make it as difficult to scale-up as ALD. Furthermore, calcination between each deposition step prevents tuning of the overcoat.

Here, we present a simple method for the liquid-phase deposition of conformal alumina overcoats. Our technique is based on the alternated exposure of the substrate's hydroxyl groups to metal oxide precursors and water. Instead of purging excess precursor, we subsequently inject stoichiometric amounts of reactants to avoid side-reactions with unreacted excess precursor. This method enables fast and straightforward cycling and overcoat deposition. We demonstrate that, like ALD, our overcoat stabilizes a supported $\mathrm{Cu} / \mathrm{Al}_{2} \mathrm{O}_{3}$ catalyst during the liquid-phase hydrogenation of biomass-derived furfural. This stabilization occurs despite the fact that the overcoat is significantly more porous than that obtained with ALD, thus retaining high copper accessibility.

\section{Experimental}

\subsection{Chemicals and materials}

All commercial chemicals were analytical reagents, and were used without further purification unless otherwise noted. Copper nitrate trihydrate (99.999-Cu\%) was purchased from ABCR. The concentrated hydrochloric acid aqueous solution (ca. 37\%) was purchased from Merck. The concentrated nitric acid aqueous solution (ca.68\%) was obtained from Sigma-Aldrich. Synthetic air (99.999\%), hydrogen (99.999\%), helium (99.9999\%), $\mathrm{N}_{2} \mathrm{O}$ (99.99\%)/helium (99.999\%) 1/99 vol./vol. and nitrogen (99.999\%) were obtained from Carbagas. Silicon carbide (100 mesh) was purchased from Strem. Furfural (99\%) was purchased from Acros organic, purified by distillation under reduced pressure and stored under inert atmosphere. Aluminum sec-butoxide (97\%) was purchased from Merck, purified by distillation under reduced pressure and stored over $4 \AA$ molecular sieves (Merck) under inert atmosphere. Alumina $\left(37 \mathrm{~m}^{2} / \mathrm{g}\right.$, Nano Arc) was purchased from Alpha Aesar and calcined for $12 \mathrm{~h}$ at $500{ }^{\circ} \mathrm{C}$ $\left(5^{\circ} \mathrm{C} / \mathrm{min}\right)$ under a flow of air $(100 \mathrm{~mL} / \mathrm{min}) .1$-butanol (99.5\%) was purchased from Acros. 2-butanol (99\%) was purchased from SigmaAldrich, dried over magnesium, distilled, stored over 4 Å molecular sieves (Merck) and degassed under vacuum. Water was purified using a Millipore Milli-Q Advantage A10 water purification system to a resistivity higher than $18 \mathrm{M} \Omega . \mathrm{cm}$.

\subsection{Catalyst preparation}

\subsubsection{Preparation of alumina-supported copper NPs by incipient} wetness impregnation

The alumina support was pretreated under vacuum $\left(<10^{-3} \mathrm{mbar}\right.$ ) at $150^{\circ} \mathrm{C}$ (heated to temperature with a $5^{\circ} \mathrm{C} / \mathrm{min}$ ramp) for $12 \mathrm{~h}$ and stored in a nitrogen filled glovebox prior to impregnation. A suitable amount of copper nitrate trihydrate was dissolved in a $0.1 \mathrm{M}$ nitric acid aqueous solution and added dropwise to the support until incipient wetness was reached. The resulting powder was dried for $12 \mathrm{~h}$ at $95^{\circ} \mathrm{C}$ in air, reduced for $5 \mathrm{~h}$ under $\mathrm{H}_{2}$ flow $\left(300^{\circ} \mathrm{C}\right.$, heated to temperature with a $1^{\circ} \mathrm{C} / \mathrm{min}$ ramp) and finally passivated at room temperature with air pulses carried by a flow of $\mathrm{N}_{2}$.

\subsubsection{Deposition of porous alumina overcoat}

The catalysts were pretreated under vacuum $\left(<10^{-3}\right.$ mbar $)$ at $300{ }^{\circ} \mathrm{C}$ for $5 \mathrm{~h}$ and kept under inert atmosphere using Schlenk techniques and a nitrogen-filled glovebox (typically $<0.5 \mathrm{ppm}_{2} \mathrm{O}$ and $<0.5 \mathrm{ppm} \mathrm{O}_{2}$ ). The powder was dispersed by magnetic stirring in anhydrous 2-butanol and the mixture was heated to $60^{\circ} \mathrm{C}$. A solution was prepared for each precursor in a Schlenk flask by dissolving the appropriate amounts of water and aluminum sec-butoxide, respectively, in 2-butanol. $0.5 \mathrm{~mL}$ of the precursor solution (corresponding to coverage by a single monolayer) were alternatively injected every $15 \mathrm{~min}$ using syringes. The coverage of a single monolayer was estimated by a volume projection of the precursor molecule. This projected area was estimated using the MarvinSketch ChemAxon software. The quantity of material needed for a single layer per catalyst surface was calculated from this calculated area for a single complex.

Typically, cycles were repeated $15,30,45$ or 60 times to obtain different overcoat thicknesses. The overcoated catalyst was separated by centrifugation $(4000 \mathrm{rpm}, 5 \mathrm{~min})$, washed twice with 2-butanol $(40 \mathrm{~mL})$ and twice with water $(40 \mathrm{~mL})$. Finally, the collected powder was dried in an oven for $12 \mathrm{~h}$ at $150^{\circ} \mathrm{C}$ (heated to temperature with a $2^{\circ} \mathrm{C} / \mathrm{min}$ ramp).

\subsection{Catalytic testing}

Furfural hydrogenation was investigated using a packed bed down flow tubular reactor (i.d $=4.5 \mathrm{~mm}$ ). Typically, $0.2 \mathrm{~g}$ of catalyst were diluted into a bed of silicon carbide within the heated zone of the reactor, which was delimited by conductive aluminum blocks placed within the oven. The reactor was leak-tested under 30 bar of nitrogen pressure.

Before the first run, the catalyst was pretreated under air flow $\left(100 \mathrm{~mL} / \mathrm{min}\right.$ ) for $1 \mathrm{~h}$ at $300^{\circ} \mathrm{C}$ (heated to temperature with a $2{ }^{\circ} \mathrm{C} / \mathrm{min}$ ramp), cooled down to $30^{\circ} \mathrm{C}$ under nitrogen flow $(100 \mathrm{~mL} / \mathrm{min})$ and reduced under $\mathrm{H}_{2}$ flow $(100 \mathrm{~mL} / \mathrm{min})$ for $5 \mathrm{~h}$ at $300^{\circ} \mathrm{C}$ (heated to temperature with a $1^{\circ} \mathrm{C} / \mathrm{min}$ ramp). After cooling down to the reaction temperature $\left(130^{\circ} \mathrm{C}\right)$, hydrogen flow was set to $35 \mathrm{~mL} / \mathrm{min}$ using a Brooks mass flow controller and pressure was adjusted to 23 bar using a Tescom back pressure regulator. A furfural solution $(70 \mathrm{~g} / \mathrm{kg}$ in 1-butanol) was then pumped through the reactor using SSI Series II HPLC pump at $0.10 \mathrm{~mL} / \mathrm{min}$. Liquid samples were collected using a gas-liquid separator. Gas phase analyses were performed on an Agilent Technologies 7890 A gas chromatography apparatus equipped with a flame ionization detector (FID) and a HP-5 column ( $50 \mathrm{~m}, 0.32 \mathrm{~mm})$.

After reaction, furfural flow was stopped and the catalyst was regenerated by calcination under a flow of air $\left(400^{\circ} \mathrm{C}, 1 \mathrm{~h}\right.$, heated to temperature with a $2{ }^{\circ} \mathrm{C} / \mathrm{min}$ ramp, $\left.100 \mathrm{~mL} / \mathrm{min}\right)$ and reduction under hydrogen flow $\left(300^{\circ} \mathrm{C}, 5 \mathrm{~h}\right.$, heated to temperature with a $1{ }^{\circ} \mathrm{C} / \mathrm{min}$ ramp, $100 \mathrm{~mL} / \mathrm{min}$ ). 


\subsection{Catalyst characterization}

\subsubsection{Inductively coupled plasma optical emission spectrometry (ICP OES)}

Elemental analyses were performed at the EPFL Central Environmental Laboratory using inductively coupled plasma optical emission spectrometry (ICP OES) ICPE-9000 multitype, Shimadzu. Metals were carefully dissolved using a mixture of concentrated nitric and hydrochloric acids (1:2 vol:vol).

\subsubsection{Transmission electron microscopy (TEM)}

The overcoat's morphology was characterized by Transmission Electron Microscopy and High-Angle Annular Dark-Field Scanning Transmission Electron Microscopy (HAADF-STEM) on a FEI Talos with a $200 \mathrm{kV}$ acceleration voltage. Lacey carbon grids were prepared by directly 'dipping' the grid into the alumina powder. The particle size distribution was estimated by statistical analysis of $c a$. 200 particles. Metal dispersion, defined as the ratio between surface and total metal atoms, was calculated back from particle size distribution assuming a spherical geometry. Elements were mapped using energy dispersive X-ray spectroscopy (EDX).

\subsubsection{Physisorption and chemisorption}

Brunauer-Emmett-Teller (BET) surfaces and Barrett-JoynerHalenda (BJH) pore volumes (desorption branch) were measured by $\mathrm{N}_{2}$-physisorption on a Micromeritics 3Flex apparatus at liquid nitrogen temperature. Samples were dried at $120^{\circ} \mathrm{C}$ under vacuum $\left(<10^{-3} \mathrm{mbar}\right)$ for $4 \mathrm{~h}$ prior to analysis.

Quantification of surface copper was performed on a Micromeritics Autochem II 2920 using $\mathrm{N}_{2} \mathrm{O}$ as a reactive probe selective to surface atoms according to the method described by Vannice et al. [22]. Typically, $0.2 \mathrm{~g}$ of sample were reduced in situ $\left(300^{\circ} \mathrm{C}, 1 \mathrm{~h}\right.$, heated to temperature with a $10^{\circ} \mathrm{C} / \mathrm{min}$ ramp) and contacted with $0.5 \mathrm{~mL} \mathrm{~N}_{2} \mathrm{O} / \mathrm{He}$ (1/99 vol./vol.) pulses using a sampling loop carried by a flow of helium at $90^{\circ} \mathrm{C}$ while $\mathrm{N}_{2}$ evolution and $\mathrm{N}_{2} \mathrm{O}$ consumption were quantified by monitoring masses 28 and 44 using MKS Cirrus 2 mass spectrometer.

\section{Results and discussion}

Alumina spheres ( $50 \mathrm{~nm}, \mathrm{~S}_{\mathrm{BET}}=34 \mathrm{~m}^{2} / \mathrm{g}$ ) were used as a support to facilitate overcoat conformity analysis by microscopy. Aluminum tri-sec-butoxide $\left(\mathrm{Al}(\mathrm{OsBu})_{3}\right)$ was selected as an aluminum source for overcoating due to its high solubility in alcohols and reactivity with water. Prior to overcoating, copper nanoparticles were formed by calcination and reduction of alumina impregnated with a solution of copper nitrate. High-Angle Annular Dark-Field Scanning Transmission Electron Microscopy (HAADF-STEM) pictures showed that the resulting nanoparticle size was $3.4 \pm 0.7 \mathrm{~nm}$ or a $32 \%$ metal dispersion assuming spherical geometry [23] (Fig. 1A, histogram Fig. S1).

Our technique is based on an accurate determination of precursor stoichiometries. Any excess precursor left in solution after reaction led to homogeneous nucleation during the subsequent half-cycle. This occurrence then created a seed for undesired heterogeneous nucleation throughout the process, which resulted in the creation of additional support, diluting the active site without any stabilization. In contrast, the addition of insufficient amounts of precursor left the surface partially uncovered, with some unprotected metal particles. Therefore, precise determination of the initial amount of precursor was essential to ensure conformal surface coverage. The support was treated at a relatively low dehydroxylation temperature $\left(300^{\circ} \mathrm{C}\right)$ in order to create a high surface hydroxyl density $\left(>6 \mathrm{OH} / \mathrm{nm}^{2}\right.$ ) [24]. In these conditions, the large size of aluminum tri-sec-precursors prevented full occupancy of surface hydroxyl groups. Therefore, monolayer coverage could be calculated assuming a surface fully packed with precursor molecules according to a projection of their van der Waals volume (Fig. 2A) (calculated as $0.65 \mathrm{~nm}^{2}$ ). The $\mathrm{Al}_{2} \mathrm{O}_{3}$ sphere surface area was measured by physisorption $\left(\mathrm{S}_{\mathrm{BET}}=34 \mathrm{~m}^{2} / \mathrm{g}\right)$ and was divided by the precursor's projected surface area to calculate a monolayer coverage stoichiometry of $87 \mu \mathrm{mol}$ of precursor per gram of $\mathrm{Al}_{2} \mathrm{O}_{3}$ spheres.

The supported catalyst was carefully suspended in anhydrous sec-butanol using sonication to avoid any diffusion transfer issues with large agglomerates. Then, coating was done by alternatively injecting $0.5 \mathrm{~mL}$ of sec-butanol solutions containing the calculated amounts of either aluminum tri-sec-butoxide or water every 15 min through an air-tight septum (Fig. 2B and C). A blank test was performed in the absence of the support to ensure that a full reaction between precursors occurred within the alternate injection time frame. We also performed a coating with a reaction time between injections of $60 \mathrm{~min}$, but this did not affect the properties of the overcoat, demonstrating that the reaction was largely over within $15 \mathrm{~min}$. After completion of the targeted number of cycles, the material was washed with sec-butanol to eliminate any unreacted precursor and with water to ensure complete hydrolysis of alkoxide ligands. The resulting substrate was then dried for storage.

STEM-HAADF revealed the presence of a low-density overcoat surrounding the dense spherical substrate after 60 cycles (Fig. 1B). Conformality did not match that achieved with ALD, but the surface was largely overcoated, as confirmed by subsequent nanoparticle characterization. The high porosity observed by TEM was confirmed by nitrogen physisorption measurements, which showed a linear increase of BET surface area from $34 \mathrm{~m}^{2} / \mathrm{g}$ for $\mathrm{Al}_{2} \mathrm{O}_{3}$ spheres to $68,80,105$ and $117 \mathrm{~m}^{2} / \mathrm{g}$ after deposition of $15,30,45$ and 60 cycles, respectively (Fig. 2D, $\mathrm{N}_{2}$ adsorption and desorption isotherms are presented in Fig. S2). Accessibility of the copper nanoparticles was assessed by titration of $\mathrm{Cu}^{0}$ sites using $\mathrm{N}_{2} \mathrm{O}$ after reduction at $300^{\circ} \mathrm{C}$. Surface copper before overcoating was $38 \mu \mathrm{mol} / \mathrm{g}$ and dropped to $21 \mu \mathrm{mol} / \mathrm{g}$ after deposition of 30 cycles confirming partial coverage of the metal. Nevertheless, about half of the surface copper was still accessible after coating, confirming the relatively high porosity of the overcoat and suggesting that the access to the metal and associated catalytic activity could be preserved. Interestingly, the number of deposition cycles has no significant influence on the $\mathrm{Cu}$ surface accessibility (19 and $23 \mu \mathrm{mol} / \mathrm{g}$ of surface $\mathrm{Cu}$ sites were measured for 15 and 50 cycles, respectively). In contrast, the dense overcoat deposited by ALD covered the metal surface completely and required calcination and cracking to restore access to the catalytic sites [10]. No further cracking or etching of the overcoat was required using the present method.

The influence of the assumed coverage stoichiometry was studied by varying the quantity of alkoxide precursor injected at each cycle. Quantities corresponding to 0.5, 1 and 2 monolayers per injection as calculated by volume projection were used in coatings with 30 cycles (Fig. 3A). The average thickness and shape irregularity of the overcoat were measured and calculated to allow for a quantitative comparison of the materials. The irregularity parameter was calculated as the standard deviation based on 10 measures of the overcoat thickness for each particle (see Section S3 for a detailed description of the calculation). A smaller irregularity value corresponds to a more conformal coating. When quantities of alkoxy precursor corresponding to 0.5 monolayers per cycle were injected, the resulting overcoat had a similar thickness to that observed when quantities corresponding to 1 monolayer were injected (Fig. 3A, Table S1). However, the irregularity in shape was significantly higher with 0.5 monolayer equivalents per cycle. This increase in irregularity is consistent with the formation of a partially coated substrate, and with subsequent cycles preferably growing on the recently formed low-density overcoat (Fig. 3A I). As expected, the use of an amount of alkoxy precursor corresponding 

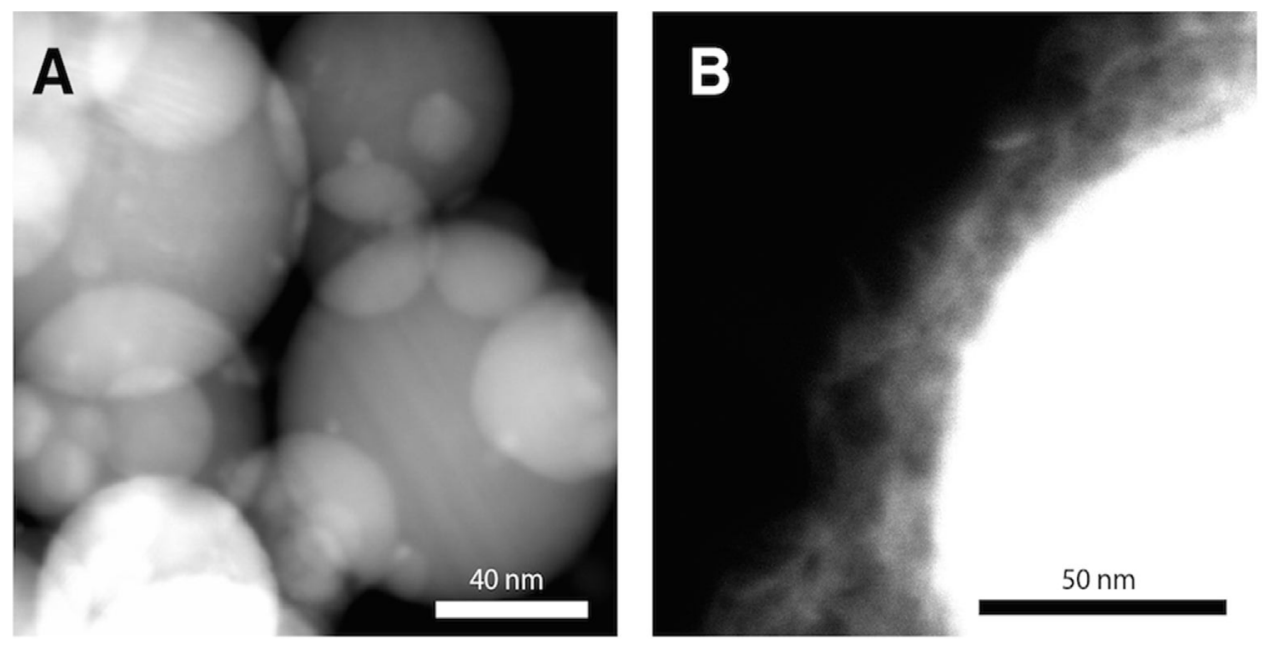

Fig. 1. STEM HAADF images of (A) Copper nanoparticles supported on alumina spheres ( $\left.\mathrm{Cu} / \mathrm{Al}_{2} \mathrm{O}_{3}\right) ;(B) \mathrm{Cu} / \mathrm{Al}_{2} \mathrm{O}_{3}$ after deposition of an amorphous alumina overcoat (60 cycles, using a water-to-aluminum molar ratio of 2.0 ).

A

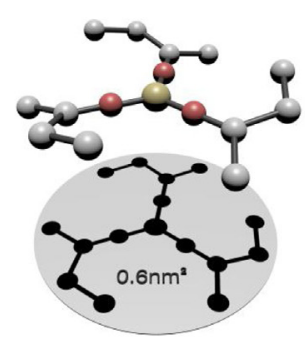

B

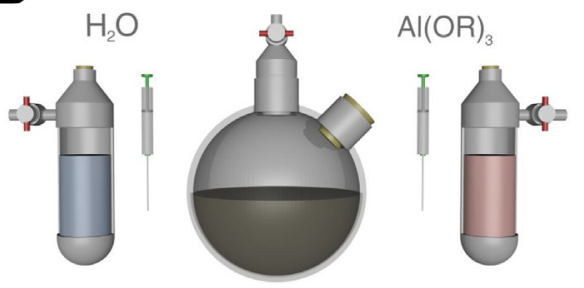

C

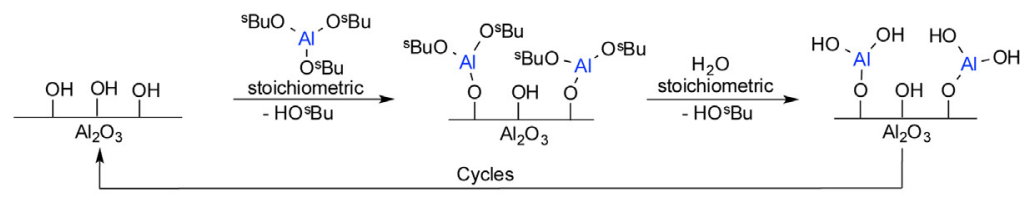

D

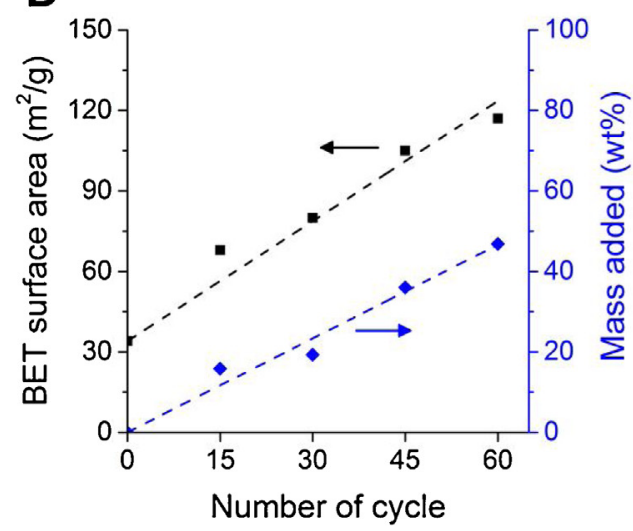

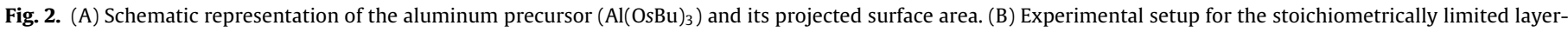

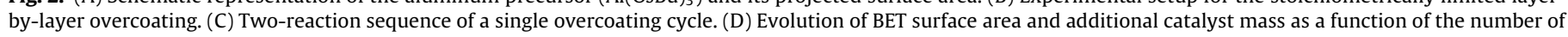
overcoating cycles (using a water to aluminum molar ratio of 2 ).

to 2 monolayers per cycle led to an average overcoat thickness that was twice as large as when 1 monolayer was used (Fig. 3A). However, the irregularity was twice as high as when a single monolayer was targeted at each cycle (Fig. 3A III). This could be explained by the deposition of large alumina clusters on the surface after they were formed in solution by homogeneous nucleation. Overall, the ideal precursor to surface stoichiometry appeared to be the amount of precursor estimated to form a single monolayer (Fig. 3A II). This result supported the assumption that this amount can be calculated using a simple volume projection of the alkoxy precursor on the surface of the support.

The reaction of an alkoxy precursor with hydroxyl groups on the surface of the support led to the formation of a population of mono, bis and tris-grafted species by substitution of 1,2 or 3 monodentate sec-butoxy ligands with surface hydroxyl groups and the associated release of sec-butanol in solution (Fig. 2C). The remaining ligands had to be hydrolyzed by the addition of water, which repopulated the surface with hydroxyl groups. Therefore, the amount of water required to hydrolyze any remaining ligands depends on the connectivity of the grafted alkoxy group. A lower amount of required water will be associated with a higher connectivity and, thus, a denser overcoat. We tested several water-to-aluminum ratios (1.0, 1.5 and $\left.2.0 \mathrm{H}_{2} \mathrm{O}: \mathrm{Al}(\mathrm{OsBu})_{3} \mathrm{~mol}: \mathrm{mol}\right)$. Using water-toaluminum ratios of 1.0 and 2.0 led to larger pore volumes (both
$0.32 \mathrm{~cm}^{3} / \mathrm{g}$, calculated according to Barrett, Joyner and Halenda $\mathrm{BJH}-$ model) and high accessibility of copper after coating (19 and $15 \mu \mathrm{mol} / \mathrm{g}$, respectively) (Fig. 3B I and III). In contrast, a denser layer $\left(0.18 \mathrm{~cm}^{3} / \mathrm{g}\right)$ preventing access to copper $(0 \mu \mathrm{mol} / \mathrm{g})$ was formed using a water-to-aluminum ratio of 1.5 (Fig. 3B II). This result suggests that aluminum atoms could react with up to an average of 1.5 surface grafted hydroxyl groups, and could thus require 1.5 water molecules to be hydrolyzed. Lower water ratios could leave non-hydrolyzed sec-butoxide ligands behind, which would act as spacers, favoring porosity (Fig. 3B I). Higher water ratios favored over-hydrolyzing the alkoxy precursor, preventing it from crosscondensing with other grafted hydroxyl groups, which could also favor increased porosity (Fig. 3B III). This proposed effect is illustrated in Fig. 3B, and demonstrates the control that sol-gel synthesis parameters can exert over the overcoat - notably, to tune the accessibility to the metallic active sites.

The catalytic performances of the uncoated and coated catalysts (60 cycles, using a water-to-aluminum molar ratio of 2.0 ) were tested in the liquid-phase hydrogenation of furfural in 1-butanol (23 bar $\mathrm{H}_{2}, 130^{\circ} \mathrm{C}$ ). Furfural is a key biomass-derived platform molecule and its transformation to furfuryl alcohol is highly relevant for biomass valorization to certain fuels and bulk chemical intermediates [6,25-27]. Recent studies have shown that furfural production is favored in organic solvents as opposed to aqueous 


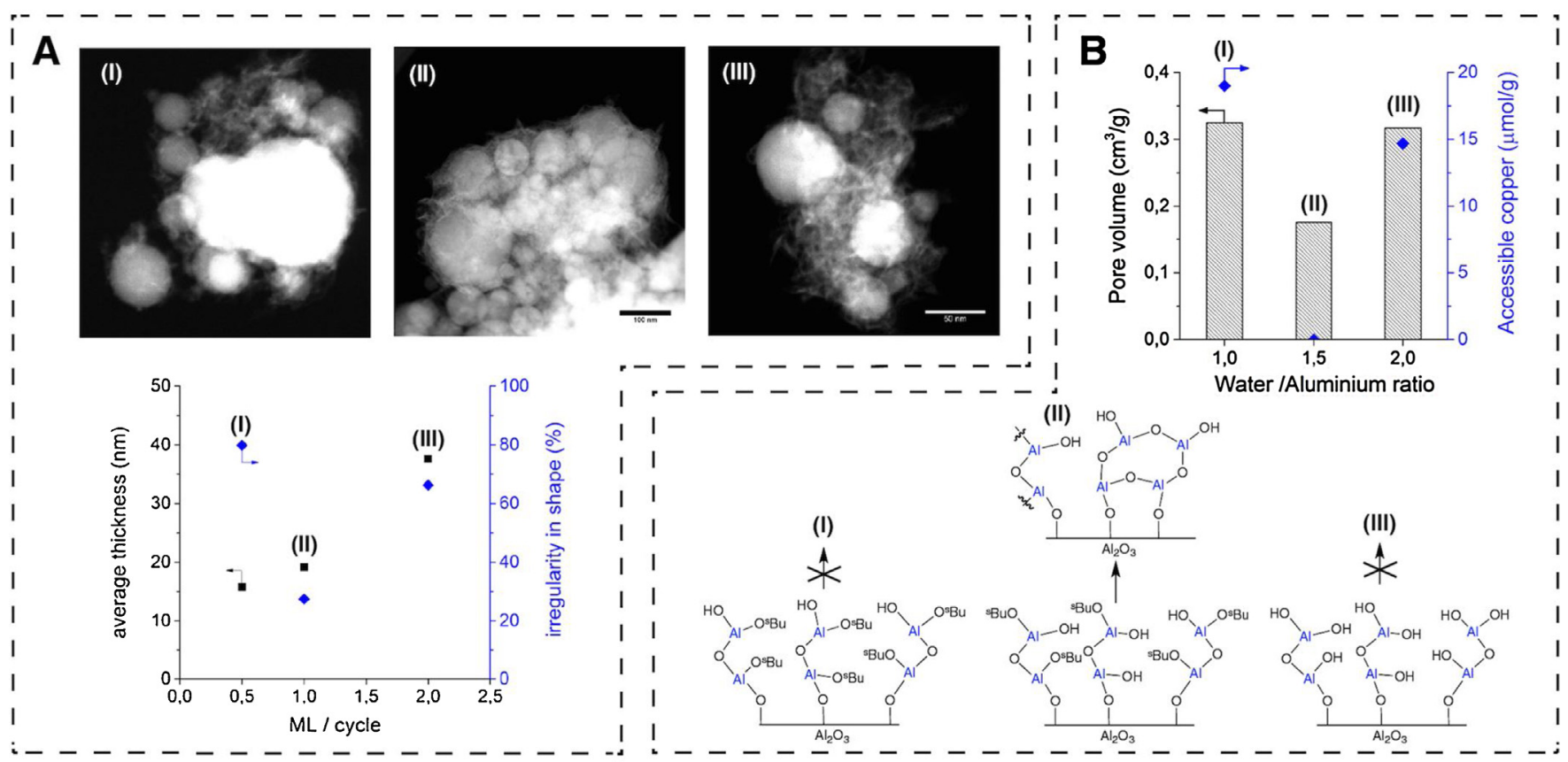

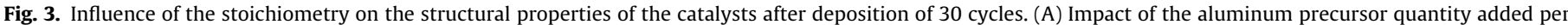

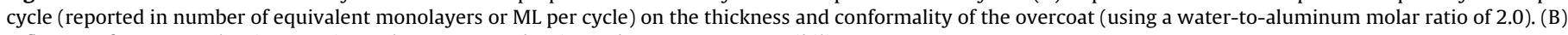
Influence of water-to-aluminum ratio on the overcoat's density and on copper's accessibility.

conditions, and is often produced at relatively dilute concentrations (i.e. $<10 \mathrm{wt} \%$ ) making its separation energy intensive $[6,25,28,29]$. Therefore, its direct conversion in the liquid phase would be both economically and energetically advantageous. However, such liquid-phase conditions can often lead to rapid irreversible deactivation of the catalyst by sintering and/or leaching.

Indeed, the uncoated catalyst rapidly deactivated with time-onstream, showing a 9-fold activity decrease over $48 \mathrm{~h}$ (Fig. 4A). Some of this activity could be recovered by regeneration of the catalyst by calcination at $400{ }^{\circ} \mathrm{C}$. This demonstrates that the deactivation was partially due to the deposition of carbonaceous residues on the surface of the catalyst. However, each regeneration cycle led to an irreversible loss of activity, until only about $16 \%$ of the initial activity was recovered after the 5th regeneration. This decrease indicated that significant irreversible deactivation had occurred over the course of several reaction/regeneration cycles. The low conversion ( $c a .7 \%, \sim 100 \%$ selectivity to furfuryl alcohol) measured at the end of run 6 equals conversion measured in the absence of copper and hydrogen, and hence could be entirely attributed to transfer hydrogenation by $\mathrm{Al}_{2} \mathrm{O}_{3}$ support (see further discussion in Section S4). Leaching of the copper in solution was not significant, as determined by inductively coupled plasma optical emission spectrometry (ICP OES) analysis of the collected samples after concentration, which showed the presence of $\sim 0.10 \%$ of the total original copper (see Table S2 for detailed leaching calculations). Mapping using energy dispersive X-ray spectroscopy (EDX) revealed the presence of large copper clusters ( $>200 \mathrm{~nm}$ ) after reaction (Fig. S3). The mean particle size was increased to $47 \pm 46 \mathrm{~nm}$ (histogram Fig. S4), corresponding to $4 \%$ metal dispersion assuming spherical geometry compared to the original $32 \%$. This 8 -fold decrease in dispersion corresponded very closely to the 6.5 -fold decrease in activity observed after 5 regeneration cycles. Therefore, the loss of activity was largely attributed to sintering, confirming previous findings [30].

When using the catalyst overcoated with 60 cycles, the initial activity was within $20 \%$ of the activity of the uncoated material, confirming the excellent accessibility of copper surface sites through the porous overcoat (Fig. 4B). In contrast, ALD overcoating with 45 cycles led to significant blockage of copper surface sites even after calcination and cracking of the dense $\mathrm{Al}_{2} \mathrm{O}_{3}$ overcoat [10]. This ALD overcoat led to a highly stable catalyst in liquid-phase conditions but also to an activity that was 10 times lower than that of the uncoated catalyst before deactivation. In fact, despite having a copper loading that was almost 3 times higher, the number of surface copper sites accessible on the ALD-overcoated was 20\% higher

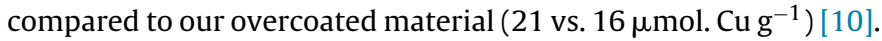

Similar to the uncoated material, catalytic activity decreased with time-on-stream (Fig. 4B). However, unlike the uncoated material, initial activity was fully recovered (and sometimes exceeded) after regeneration by calcination, demonstrating that only reversible deactivation had occurred (Fig. 4B). This stable activity was maintained for 6 reaction runs and 5 regeneration cycles over the course of a 3-week period, showing that irreversible loss of metal surface area is suppressed by the presence of the stoichiometrically deposited overcoat. No leaching $(\sim 0.15 \%$ total $\mathrm{Cu}$, see table S2) was detected, while sintering of copper particles was negligible. The contrast between small copper particles and alumina in microscopy was poor for overcoated samples due to the small density differences between the support, metal particles, and overcoat. This prevented an accurate determination of the metal dispersion by microscopy before and after catalysis. However, the large particles $(>10 \mathrm{~nm}$ ) that were observed after reaction in the uncoated catalyst (Fig. S4) would have been visible by EDX mapping despite these imaging limitations for the overcoated catalyst. However, no such large particles were observed, further confirming that sintering had been successfully curtailed.

\section{Conclusions}

The effective stabilization of the catalyst indicates that the stoichiometrically limited liquid-phase deposition of alumina could be an effective alternative to ALD for catalyst overcoating applications. The use of stoichiometrically limited deposition allowed us to overcome the difficulties of depositing alumina in a controlled 

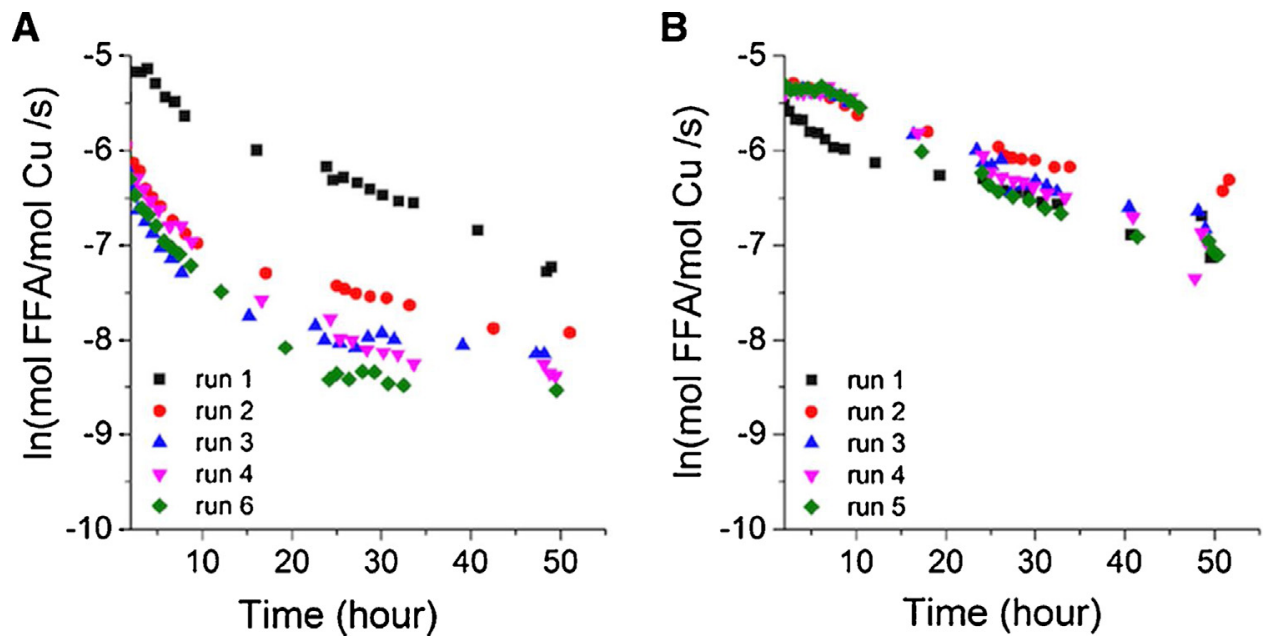

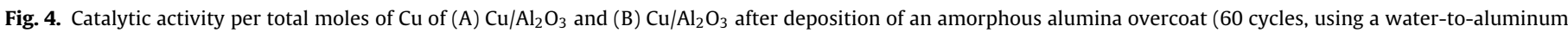

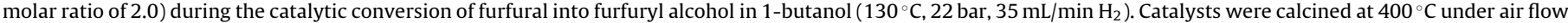
and reduced at $300{ }^{\circ} \mathrm{C}$ under $\mathrm{H}_{2}$ flow between each run.

fashion in the absence of excessive precursor purging or washing. In addition, this method is particularly sensitive to synthesis parameters, which can be changed to control the overcoat's porosity and access to the metal site. This particular control over the overcoat's nanoarchitecture is absent in gas phase methods and could facilitate further tailoring of the active site's nanoscale environment. Efforts to use these properties to control the catalyst's activity and selectivity are currently underway.

\section{Acknowledgements}

We thank the EPFL interdisciplinary center for electron microscopy for support during electron microscopy measurements. We acknowledge funding from the Swiss National Science Foundation through grant PYAPP2_154281; and by EPFL. K.B. was supported by funding from the James B. Reynolds Scholarship for Foreign Study through Dartmouth University.

\section{Appendix A. Supplementary data}

Supplementary data associated with this article can be found, in the online version, at http://dx.doi.org/10.1016/j.apcatb.2017. 07.006.

\section{References}

[1] J.-P. Lange, Renewable feedstocks: the problem of catalyst deactivation and its mitigation, Angew. Chem. Int. Ed. 54 (2015) 13186-13197.

[2] C.H. Bartholomew, Mechanisms of catalyst deactivation, Appl. Catal. A 212 (2001) 17-60.

[3] T.W. Hansen, A.T. DeLaRiva, S.R. Challa, A.K. Datye, Sintering of catalytic nanoparticles: particle migration or ostwald ripening? Acc. Chem. Res. 46 (2013) 1720-1730.

[4] F. Héroguel, B. Rozmysłowicz, J.S. Luterbacher, Improving heterogeneous catalyst stability for liquid-phase biomass conversion and reforming, Chim. Int. J. Chem. 69 (2015) 582-591.

[5] T.J. Schwartz, B.J. O'Neill, B.H. Shanks, J.A. Dumesic, Bridging the chemical and biological catalysis gap: challenges and outlooks for producing sustainable chemicals, ACS Catal. 4 (2014) 2060-2069.

[6] J.S. Luterbacher, D.M. Alonso, J.A. Dumesic, Targeted chemical upgrading of lignocellulosic biomass to platform molecules, Green Chem. 16 (2014) 4816-4838.

[7] M.V. Twigg, M.S. Spencer, Deactivation of supported copper metal catalysts for hydrogenation reactions, Appl. Catal. A 212 (2001) 161-174.

[8] H. Yi, H. Du, Y. Hu, H. Yan, H.-L. Jiang, J. Lu, Precisely controlled porous alumina overcoating on Pd catalyst by atomic layer deposition: enhanced selectivity and durability in hydrogenation of 1,3-Butadiene, ACS Catal. 5 (2015) 2735-2739.
[9] J. Lu, B. Fu, M.C. Kung, G. Xiao, J.W. Elam, H.H. Kung, P.C. Stair, Coking- and sintering-resistant palladium catalysts achieved through atomic layer deposition, Science 335 (2012) 1205-1208.

[10] B.J. O’Neill, D.H.K. Jackson, A.J. Crisci, C.A. Farberow, F. Shi, A.C. Alba-Rubio, J. Lu, P.J. Dietrich, X. Gu, C.L. Marshall, P.C. Stair, J.W. Elam, J.T. Miller, F.H. Ribeiro, P.M. Voyles, J. Greeley, M. Mavrikakis, S.L. Scott, T.F. Kuech, J.A. Dumesic, Stabilization of copper catalysts for liquid-phase reactions by atomic layer deposition, Angew. Chem. Int. Ed. 52 (2013) 13808-13812.

[11] J.W. Han, C. Kim, J.S. Park, H. Lee, Highly coke-pesistant Ni nanoparticle catalysts with minimal sintering in dry reforming of methane, ChemSusChem 7 (2014) 451-456.

[12] I. Lee, Q. Zhang, J. Ge, Y. Yin, F. Zaera, Encapsulation of supported Pt nanoparticles with mesoporous silica for increased catalyst stability, Nano Res. 4 (2010) 115-123.

[13] Z. Bo, T.R. Eaton, J.R. Gallagher, C.P. Canlas, J.T. Miller, J.M. Notestein, Size-selective synthesis and stabilization of small silver nanoparticles on TiO2 partially masked by SiO2, Chem. Mater. 27 (2015) 1269-1277.

[14] M. Caillot, A. Chaumonnot, M. Digne, C. Poleunis, D.P. Debecker, J.A. vanBokhoven, Synthesis of amorphous aluminosilicates by grafting: tuning the building and final structure of the deposit by selecting the appropriate synthesis conditions, Micropor. Mesopor. Mater. 185 (2014) 179-189.

[15] F. Héroguel, G. Siddiqi, M.D. Detwiler, D.Y. Zemlyanov, O. Safonova, C. Copéret, Simultaneous generation of mild acidic functionalities and small supported Ir NPs from alumina-supported well-defined iridium siloxide, J. Catal. 321 (2015) 81-89.

[16] K. Wang, Y. Lin, M.A. Morris, J.D. Holmes, Preparation of MCM-48 materials with enhanced hydrothermal stability, J. Mater. Chem. 16 (2006) 4051-4057.

[17] F. Zhang, X. Carrier, J.-M. Krafft, Y. Yoshimura, J. Blanchard, Insight into the structure and localization of the titania overlayer in TiO2-coated SBA-15 materials, New J. Chem. 34 (2010) 508-516.

[18] E. Santacesaria, M. Cozzolino, M. Di Serio, A.M. Venezia, R. Tesser, Vanadium based catalysts prepared by grafting: preparation, properties and performances in the ODH of butane, Appl. Catal. A 270 (2004) 177-192.

[19] W. Yan, S.M. Mahurin, S.H. Overbury, S. Dai, Nanoengineering catalyst supports via layer-by-layer surface functionalization, Top. Catal. 39 (2006) $199-212$.

[20] M. Baca, E. de la Rochefoucauld, E. Ambroise, J.-M. Krafft, R. Hajjar, P.P. Man, X. Carrier, J. Blanchard, Characterization of mesoporous alumina prepared by surface alumination of SBA-15, Micropor. Mesopor. Mat. 110 (2008) 232-241.

[21] P. Iengo, M. Di Serio, A. Sorrentino, V. Solinas, E. Santacesaria, Preparation and properties of new acid catalysts obtained by grafting alkoxides and derivatives on the most common supports note I - grafting aluminium and zirconium alkoxides and related sulphates on silica, Appl. Catal. A 167 (1998) 85-101.

[22] A. Dandekar, M.A. Vannice, Determination of the dispersion and surface oxidation states of supported Cu catalysts, J. Catal. 178 (1998) 621-639.

[23] K. Larmier, S. Tada, A. Comas-Vives, C. Copéret, Surface sites in Cu-nanoparticles: chemical reactivity or microscopy? J. Phys. Chem. Lett. 7 (2016) 3259-3263.

[24] R. Wischert, C. Coperet, F. Delbecq, P. Sautet, Optimal water coverage on alumina: a key to generate lewis acid-base pairs that are reactive towards the C-H bond activation of methane, Angew. Chem. Int. Ed. 50 (2011) 3202-3205.

[25] D.M. Alonso, S.G. Wettstein, M.A. Mellmer, E.I. Gurbuz, J.A. Dumesic, Integrated conversion of hemicellulose and cellulose from lignocellulosic biomass, Energy Environ. Sci. 6 (2012) 76-80.

[26] C.M. Cai, T. Zhang, R. Kumar, C.E. Wyman, Integrated furfural production as a renewable fuel and chemical platform from lignocellulosic biomass, J. Chem. Technol. Biotechnol. 89 (2014) 2-10. 
[27] A. Eseyin, E.E. Anthonia, H.S. Philip, An overview of the applications of furfural and its derivatives, IJAC 3 (2015) 42-47.

[28] M.A. Mellmer, C. Sener, J.M.R. Gallo, J.S. Luterbacher, D.M. Alonso, J.A.

Dumesic, Solvent effects in acid-catalyzed biomass conversion reactions, Angew. Chem. Int. Ed. 53 (2014) 11872-11875.
[29] L. Shuai, J. Luterbacher, Organic solvent effects in biomass conversion reactions, ChemSusChem 9 (2016) 133-155.

[30] B.J. O’Neill, J.T. Miller, P.J. Dietrich, F.G. Sollberger, F.H. Ribeiro, J.A. Dumesic Operando X-ray absorption spectroscopy studies of sintering for supported copper catalysts during liquid-phase reaction, ChemCatChem 6 (2014) 2493-2496. 